\title{
Pregnancy Outcomes During the Clinical Development Program of Cladribine in Multiple Sclerosis: An Integrated Analysis of Safety
}

\author{
Gavin Giovannoni ${ }^{1} \cdot$ Andrew Galazka $^{2} \cdot$ Regina Schick $^{3} \cdot$ Thomas Leist $^{4} \cdot$ Giancarlo Comi $^{5} \cdot$ Xavier Montalban $^{6,7}$. \\ Doris Damian $^{8} \cdot$ Fernando Dangond $^{8} \cdot$ Stuart Cook $^{9}$
}

Published online: 23 May 2020

(c) The Author(s) 2020

\begin{abstract}
Introduction Although use of contraception was pre-specified during cladribine clinical trials for multiple sclerosis, some pregnancies did occur.

Objective This analysis reports on pregnancy outcomes in the cladribine clinical development program.

Methods Pregnancy outcomes in female patients (direct pregnancies) and those arising from partner pregnancies (i.e., female partners of male study participants with multiple sclerosis) were evaluated from an integrated safety analysis of ten studies of cladribine in multiple sclerosis (nine clinical trials and a long-term safety registry), with patients treated with cladribine tablets, parenteral cladribine, or placebo (all-exposed cohort; 1976 patients received cladribine and 802 received placebo). Pregnancies that occurred during the 'at-risk' period for cladribine (during treatment or within 6 months thereafter) are reported as a separate group.

Results In the all-exposed cohort, 70 direct pregnancies occurred among 62 female patients (cladribine, $n=49$; placebo, $n=21$ ). Pregnancy outcomes were: live births (cladribine, $n=19$ [38.8\%]; placebo, $n=9$ [42.9\%]), elective terminations (cladribine, $n=14$ [28.6\%]; placebo, $n=4$ [19.0\%]), spontaneous abortions (cladribine, $n=11$ [22.4\%]; placebo, $n=5$ [23.8\%]), and therapeutic terminations (cladribine, $n=5[10.2 \%]$; placebo, $n=2$ [9.5\%]); in the remaining placebo recipient, the pregnancy outcome was unknown. There were two reports of congenital malformations (cladribine, $n=1$; placebo, $n=1$ ), both of which occurred with pregnancies arising $>2$ years after exposure to the last dose of study medication. Sixteen direct pregnancies occurred during the 'at-risk' period for cladribine; outcomes for these were: live births, $n=3(18.8 \%)$; elective terminations, $n=10$ (62.5\%); spontaneous abortions, $n=2$ (12.5\%); and therapeutic terminations, $n=1(6.2 \%)$. Corresponding findings for direct pregnancies among placebo recipients were $(n=11)$ : live births, $n=5$ (45.5\%); elective terminations, $n=2(18.2 \%)$; spontaneous abortions, $n=3(27.3 \%)$; and unknown, $n=1(9.1 \%)$. No cases of congenital malformation were reported for pregnancies during the 'at-risk' period. There were an additional nine partner pregnancies in female partners of cladribine-treated male patients, all of which resulted in live births; of these, two pregnancies occurred within the 'at-risk' period for cladribine.

Conclusions While limited by the small number of pregnancies and related data from the cladribine clinical development program, highlighting the need for further study, the observations made in the present analysis were generally consistent with epidemiological data on pregnancy outcomes for the general population or women with multiple sclerosis. There were no congenital malformations in pregnancies that occurred during cladribine treatment or within 6 months after the last dose. As the data available for cladribine-exposed pregnancies in patients with multiple sclerosis are limited, a non-interventional post-authorization safety study has been initiated to obtain more information on this subject.

Clinical Trial Registration CLARITY: NCT00213135; CLARITY Extension: NCT00641537; ORACLE MS: NCT00725985; ONWARD: NCT00436826; PREMIERE: NCT01013350.
\end{abstract}

Extended author information available on the last page of the article 


\section{Key Points}

In ten clinical studies of cladribine, used to treat multiple sclerosis (MS), study subjects were asked to use contraception to avoid getting pregnant. However, some women did become pregnant, as did the female partners of some men with MS who took part in the studies. We report the outcomes for these pregnancies as part of a large safety analysis.

There were 70 pregnancies in total; 49 in the cladribine group and 21 in the placebo group. The outcomes for these pregnancies (including abortions and live births) were very similar for the two groups.

These findings are what we would expect for women in the general population, as well as women with MS who become pregnant. A new study is being conducted to obtain more information on pregnancies when women are treated with cladribine for MS.

\section{Introduction}

Multiple sclerosis (MS) is most commonly diagnosed in individuals aged 20-40 years. Three quarters of newly diagnosed cases are in women, often of childbearing age [1]. Consequently, reproductive considerations are important for female patients with MS receiving treatment with disease-modifying drugs (DMDs) that have pregnancy-related warnings.

The majority of pharmacological agents for MS require regular dosing and presence of the agent to maintain the clinical effect. This may represent a challenge to disease management in patients who wish to become pregnant, but do not wish to be at risk for MS disease activity. More recently, some treatment approaches approved or considered for MS, including autologous bone marrow transplant, alemtuzumab, ocrelizumab, and cladribine tablets, harbor the promise of longer term remission of MS disease activity beyond presence of the DMD. While the practice surrounding DMDs and pregnancy is evolving, more evidence is needed, especially for newer agents [2].

In patients with relapsing MS who received cladribine tablets $10 \mathrm{mg}$ (3.5- $\mathrm{mg} / \mathrm{kg}$ cumulative dose over 2 years, administered as one treatment course of $1.75 \mathrm{mg} / \mathrm{kg}$ per year, with each treatment course consisting of up to 10 treatment days), attenuation of disease activity was observed [3] and sustained for many patients in years 3 and 4 without further treatment [4]. Cladribine is a prodrug that enters the cell via nucleoside transporter proteins and becomes active upon phosphorylation to its 2-chlorodeoxyadenosine triphosphate metabolite. Intracellular phosphorylation of cladribine is specifically mediated by the enzyme deoxycytidine kinase and de-phosphorylation occurs due to 5'-nucleotidase [5]. The phosphorylated form preferentially accumulates in lymphocytes owing to their unique constitutively high deoxycytidine kinase levels and low 5'-nucleotidase levels compared with other cells in the body. Following activation of the drug in lymphocytes, 2-chlorodeoxyadenosine triphosphate may be incorporated into the DNA strands resulting in breaks and interference with DNA synthesis, leading to their selective apoptosis. In other cell-types, 5'-nucleotidases balance the activity of deoxycytidine kinase and prevent 2-chlorodeoxyadenosine triphosphate accumulation.

In reproductive toxicology studies performed in animals (unpublished observations, Merck KGaA), cladribine did not show effects on female fertility or on peri-postnatal development of the offspring. Cladribine was found to be teratogenic in mice and rabbits when given intravenously. In mice, cladribine did not show effects on male fertility; however, it did show depletion of germ cells, spermatids, and spermatozoa. No effects on male reproductive performance were seen as assessed after the completion of one spermatogenic cycle without cladribine treatment, and no testicular histopathological changes were seen after this one spermatogenic cycle, together demonstrating a complete recovery.

Cladribine has a short half-life in humans $(<24 \mathrm{~h})$ and after administration is rapidly eliminated from the body [6]. However, in view of its teratogenicity in mice and rabbits at doses higher than those used in humans, and the short-term effects on male germ cells noted above, caution should be exercised with respect to conception during and after cladribine dosing. Bearing in mind physiological development of reproductive tissues, 3 months after exposure to cladribine is adequate time for a follicular or spermatogenic development cycle to complete. At the time of development of prescribing information concerning the use of parenteral cladribine in hematological malignancies, 3 additional months were added as a precaution; thus, a 6-month period was proposed during which female and male patients should not conceive to avoid risk of exposure of a conceptus [7]. Following discussions with regulatory authorities at the time of approval of cladribine tablets in MS, it was decided to apply the same precautionary 6-month period. As per the prescribing information, pregnancy has to be excluded prior to starting cladribine tablets, and both female and male patients must use effective contraception during treatment and for 6 months after the last dose of cladribine to avoid pregnancy (and partner pregnancy) during this time [5]. We report on pregnancy outcomes in female patients with MS and the partners of male patients with MS who became pregnant during the cladribine clinical development program. 


\section{Methods}

\subsection{Study Population}

Pregnancy outcomes were evaluated as part of an integrated safety analysis of ten studies, including nine clinical trials and a long-term safety registry (Table 1). The analysis included patients who participated in the cladribine tablet clinical trial program in patients with a first clinical demyelinating event (i.e., CLARITY [3], CLARITY Extension [4] in patients with relapsing-remitting MS, and ORACLE-MS [9]), and the phase II ONWARD study [10], which assessed the effects of treatment with cladribine tablets plus interferon (IFN)- $\beta$ in patients with relapsing MS. The long-term safety registry (PREMIERE) followed patients from the cladribine tablet studies to provide insight into the medium-to-longer term safety of treatment with cladribine tablets in patients with relapsing MS or a first clinical demyelinating event. Patient safety data collected by the registry up to the completion date of 25 October, 2018 were included in this analysis. Patients with MS who participated in five earlier clinical studies that assessed the efficacy and safety of parenteral cladribine were also included in the analysis. The integration of safety data from all ten of these studies comprised a population of 2778 patients (cladribine, $n=1976$; placebo, $n=802$ ) recruited from over 35 countries worldwide, and represented data from the complete clinical development program for cladribine in MS. This population is referred to as the all-exposed cohort throughout this article [8].

For ethical reasons, some trials used a switch design to prevent patients initially randomized to placebo from spending prolonged periods without active treatment. In patients initially treated with placebo and subsequently with cladribine, the first 2 years of their data were analyzed as part of the placebo group and if/when they switched to cladribine, all of their subsequent data were attributed to the cladribine group. If a patient received cladribine followed by placebo, all of their data were attributed to the cladribine group; these data were not included in the placebo group analysis.

\subsection{Pregnancy Outcomes and Data Analysis}

This analysis is based on data collected through pregnancy report forms and parent child/fetus adverse event report forms used in the clinical trials that comprised the cladribine clinical development program. We first evaluated pregnancy outcomes for pregnant women with MS who were exposed to cladribine or placebo, i.e., direct pregnancies for the allexposed cohort. Thereafter, we evaluated direct pregnancies among female patients in the 'at-risk' cohort, i.e., the subgroup of patients who became pregnant either during cladribine administration or within 6 months (183 days) of the last dose (this precautionary period is specified in the prescribing information to avoid any possible embryofetal exposure to cladribine). Data were also described for partner pregnancies (i.e., those that occurred in female partners of male study participants with MS), using the same definitions of exposure outlined above.

Pregnancy outcomes were separated into the following categories: live birth, elective termination (i.e., pregnancy terminated according to the patient's decision), spontaneous abortion, therapeutic termination (i.e., medically indicated termination) with reasons, and unknown outcome. Any congenital malformations were also to be reported. According
Table 1 Summary of clinical studies using cladribine tablets or parenteral cladribine used in the integrated safety analysis

\begin{tabular}{llllr}
\hline Study & Design & Formulation & Phase & Number $^{\text {a }}$ \\
\hline CLARITY [3] & R, DB & CT & III & 1326 \\
CLARITY Extension [4] & R, DB & CT & IIIb & 806 \\
ORACLE-MS [9] & R, DB & CT & III & 617 \\
ONWARD [10] & R, DB & CT & IIb & 172 \\
PREMIERE & Extension & CT & Observational & 1170 \\
Scripps-A & OL & iv & II & 7 \\
Scripps-B & R, DB & sc & II & 11 \\
Scripps-C & R, DB & sc & II & 52 \\
MS-Scripps & R, DB & iv & II & 49 \\
MS-001 & R, DB & sc & III & 159 \\
\hline
\end{tabular}

$C T$ cladribine tablets, $D B$ double-blind, $i v$ intravenous, $M S$ multiple sclerosis, $O L$ open-label, $R$ randomized, $s c$ subcutaneous

${ }^{a}$ Number of patients randomized to double-blind treatment or enrolled into the study

${ }^{\mathrm{b}}$ The PREMIERE (Prospective Observational Long-term Safety Registry of Multiple Sclerosis Patients Who Have Participated in Cladribine Clinical Trials) registry was a prospective, observational, long-term safety study of patients with MS that commenced in 2009 and completed in 2018. It was open to patients who had participated in one of the clinical studies of cladribine tablets (CLARITY, CLARITY Extension, ORACLE-MS, or ONWARD) in which patients continued to be followed up for long-term monitoring 
to routine processes, live births were not followed beyond delivery. Follow-up was only conducted for cases with a pending pregnancy outcome, unknown pregnancy outcome, or in the case of an abnormality at birth (such as congenital anomalies or other serious health conditions) until confirmation of the abnormality or until 1 year after birth. Descriptive statistics, including number of outcomes $(n)$ and proportions, were calculated for each pregnancy outcome.

\section{Results}

\subsection{Patient Characteristics}

The all-exposed cohort consisted of 1976 patients (Table 2) who had been treated with cladribine, with a total exposure time of 9855 patient-years (1306 women, 66.1\%), and 802 patients who had received placebo for 2782 patient-years of exposure (535 women, 66.7\%). The patient-years of exposure to cladribine in the all-exposed cohort was higher than for placebo because the majority of clinical studies involved randomization of patients to treatment in a 2:1 ratio (active treatment:placebo), the possibility of switching to cladribine tablets during CLARITY Extension, and also because of the attribution policy (see also the earlier section on study population for details).

\subsection{Direct Pregnancies in Female Study Participants}

In total, 70 direct pregnancies occurred among 62 female patients in the all-exposed cohort (including repeat pregnancies during the observational registry study) (Table 3 ). In female patients who had been exposed to cladribine $(n=1306), 49$ pregnancies occurred in 43 patients. Of these, 19 of 49 (38.8\%) pregnancies resulted in live births. A pregnancy that occurred 2 years and 3 months after the last dose of cladribine tablets resulted in the birth of twins. A congenital malformation (omphalocele) was reported in one of the twins. The patient had received a cumulative dose of $3.87-\mathrm{mg} / \mathrm{kg}$ cladribine tablets in the CLARITY trial. In 14 of 49 (28.6\%) pregnancies, elective termination was selected, and in 11 of 49 (22.4\%) pregnancies, spontaneous abortions occurred. A total of five therapeutic terminations of pregnancy were performed in four patients who had received cladribine treatment (two terminations for ectopic pregnancies in one patient, a termination for choriocarcinoma in one patient, and two terminations due to fetal death in PREMIERE patients who had the last dose of cladribine approximately 3 years prior to pregnancy).

In female patients who had received placebo $(n=535)$, there were 21 direct pregnancies in 19 patients; nine of 21 (42.9\%) pregnancies led to live births, four $(19.0 \%)$ ended in elective terminations, and five (23.8\%) resulted in spontaneous abortions. Therapeutic terminations were performed for two placebo recipients, including one patient after an ultrasound scan revealed a Dandy-Walker congenital malformation and progressive placental abruption, which occurred approximately 2 years and 1 month after the last administration of placebo. The other was in a placebo recipient who experienced rupture of the amniotic sac and fetal death in the 17 th week of gestation. The outcome of pregnancy in one placebo recipient was unknown.

\subsection{Direct Pregnancies During Cladribine Dosing or Within 6 Months After the Last Dose ('At-Risk' Cohort)}

A total of 16 direct pregnancies in 16 female patients (Table 3) occurred during the period of cladribine dosing or within 6 months after the last dose; three of $16(18.8 \%)$ pregnancies were live births, ten $(62.5 \%)$ pregnancies ended in elective terminations, and two (12.5\%) ended with spontaneous abortions. Therapeutic termination for an ectopic pregnancy occurred in one patient. There were no pregnancies with congenital anomalies or with unknown outcomes.

In female patients receiving placebo, 11 pregnancies occurred of which five (45.5\%) resulted in live births. Of the 11 pregnancies, there were three (27.3\%) spontaneous
Table 2 Demographics and characteristics of patients included in the all-exposed cohort

\begin{tabular}{lll}
\hline Patient characteristics & Placebo $(n=802)$ & Cladribine $(n=1976)$ \\
\hline Female, $n(\%)$ & $535(66.7)$ & $1306(66.1)$ \\
Time on study in weeks, mean (SD) & $180.99(135.03)$ & $260.23(139.20)$ \\
Patient-years of exposure & 2782 & 9855 \\
Age at clinical study baseline (years), mean (SD) & $37.6(9.8)$ & $37.7(10.1)$ \\
Median & 37.0 & 38.0 \\
Minimum; maximum & $18 ; 64$ & $18 ; 65$ \\
Age $\leq 40$ years, $n(\%)$ & $485(60.5)$ & $1162(58.8)$ \\
Prior treatment with DMD, $n(\%)$ & $188(23.4)$ & $505(25.6)$ \\
Disease duration in years, mean (SD) & $9.50(7.44)$ & $8.91(7.21)$ \\
\hline
\end{tabular}

$D M D$ disease-modifying drug, $S D$ standard deviation 
Table 3 Pregnancy outcomes in the cladribine multiple sclerosis clinical program (direct pregnancies only)

\begin{tabular}{|c|c|c|c|c|}
\hline \multirow[t]{2}{*}{ Pregnancy outcomes, $n(\%)$} & \multicolumn{2}{|c|}{ All-exposed cohort } & \multicolumn{2}{|c|}{ At-risk cohort ${ }^{\mathrm{a}}$} \\
\hline & $\begin{array}{l}\text { Cladribine } \\
\text { pregnancies } \\
(N=49)\end{array}$ & $\begin{array}{l}\text { Placebo } \\
\text { pregnancies } \\
(N=21)\end{array}$ & $\begin{array}{l}\text { Cladribine } \\
\text { pregnancies } \\
(N=16)\end{array}$ & $\begin{array}{l}\text { Placebo } \\
\text { pregnancies } \\
(N=11)\end{array}$ \\
\hline Live birth & $19(38.8)$ & $9(42.9)$ & $3(18.8)$ & $5(45.5)$ \\
\hline Elective termination & $14(28.6)$ & $4(19.0)$ & $10(62.5)$ & $2(18.2)$ \\
\hline Spontaneous abortion & $11(22.4)$ & $5(23.8)$ & $2(12.5)$ & $3(27.3)$ \\
\hline Therapeutic termination & $5(10.2)$ & $2(9.5)$ & $1(6.2)$ & $0(0)$ \\
\hline Congenital abnormalities & $n=1^{\mathrm{b}}$ & $n=1^{\mathrm{c}}$ & 0 & 0 \\
\hline Unknown & $0(0)$ & $1(4.8)$ & $0(0)$ & $1(9.1)$ \\
\hline
\end{tabular}

Please refer to the main text for a description of outcomes for partner pregnancies (i.e., pregnancies that occurred in female partners of male study participants)

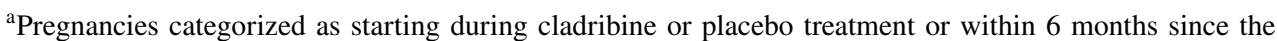
most recent dose, and are a subset of the total number of pregnancies that occurred in cladribine- or placebo-treated patients

${ }^{\mathrm{b}}$ One case of omphalocele reported in one twin from a pregnancy that occurred approximately 2 years and 3 months after the last dose of cladribine tablets

${ }^{\mathrm{c}}$ An ultrasound scan revealed a Dandy-Walker malformation in a pregnancy that occurred approximately 2 years and 1 month after the last dose of placebo abortions and two (18.2\%) elective terminations. There was one $(9.1 \%)$ pregnancy with an unknown outcome.

\subsection{Outcome of Partner Pregnancies}

For the all-exposed cohort, nine pregnancies occurred in the female partners of nine cladribine-treated male patients with MS who participated in the clinical trial program; each of these pregnancies resulted in live births, with one fetal distress recorded due to nuchal cord. There were two pregnancies among the female partners of two placebo-treated male patients with MS, and the outcomes of these were unknown.

Pregnancies occurred in two female partners of male patients treated with cladribine during the 'at-risk' period of treatment or within 6 months after the most recent dose. Both of these pregnancies resulted in live births.

\section{Discussion}

Despite the guidance to study participants about the need for contraception in each of the clinical trials, a number of pregnancies occurred in the cladribine clinical program in MS. Of the 70 pregnancies that occurred in the all-exposed cohort, $40 \%$ resulted in live births, $26 \%$ in elective terminations, $23 \%$ in spontaneous abortions, and $10 \%$ in therapeutic terminations ( $1 \%$ had an unknown outcome). Compared with placebo recipients, there was a higher proportion of termination among cladribine-treated patients. This was as expected because patients received cladribine in an openlabel manner in several studies and extension periods, and the study protocols made specific reference to the potential embryofetal risks of cladribine exposure with recommendations and measures to avoid pregnancy.

There were two reports of congenital malformations in the present analysis, one in a twin from a pregnancy arising $>2$ years after the last dose of cladribine tablets (omphalocele) and one from a pregnancy arising $>2$ years after the last dose of placebo (Dandy-Walker malformation). The omphalocele case could not be plausibly related to cladribine treatment because it occurred so long after the last dose that there was no embryofetal exposure to the drug.

The most relevant information from the present analysis concerns the pregnancy outcomes of the 16 female patients who were exposed to cladribine during the 'at-risk' period (i.e., the time window of cladribine treatment and up to 6 months after the last dose). Approximately one tenth of such pregnancies resulted in spontaneous abortions. There were no reports of congenital malformations during the 'atrisk' period. Of the three live births from these 16 pregnancies, one pregnancy occurred 2 weeks after the last dose of cladribine and two pregnancies occurred 5-6 months after the last dose. Another two live births resulted from partner pregnancies that occurred in women whose male partners had received treatment with cladribine (within 6 months of the last dose). All five of these live births resulted in otherwise healthy newborns.

While the findings of the current analysis are limited by the small sample size (which is unsurprising given that patients were advised to avoid pregnancy by using effective contraception while in the clinical trials [5]), the observations are generally consistent with epidemiological data on pregnancy outcomes for the general population or women with MS. For example, in the all-exposed and 'at-risk' 
cohorts, respectively, $22.4 \%$ and $12.5 \%$ of pregnancies in patients who were treated with cladribine ended with spontaneous abortions. This is comparable to the findings of a meta-analysis that reported on pregnancy outcomes for 13,144 women with MS, and found that the combined rate of spontaneous abortion and therapeutic termination was $27.9 \%$ (95\% confidence interval 23.6-32.7\%) [14]. Spontaneous abortions occurred in $23.8 \%$ of the placebo cohort, a proportion that is consistent with epidemiological data for the MS untreated population $[15,16]$.

Our results for partner pregnancies support previously published pregnancy data for female partners of male patients treated for MS. Reporting prospective observational data from the Italian Pregnancy Dataset, for example, Pecori et al. reported no association between DMD exposure of fathers at the time of conception and the risk of spontaneous abortion, adverse fetal outcomes, and congenital malformations; the study summarized outcomes for 78 pregnancies fathered by patients with MS, 45 (58\%) of whom were receiving DMDs at the time of conception (predominantly $\beta$-interferons $[n=39]$ and glatiramer acetate $[n=6])$ [11]. Although available data are limited, it has also been reported that MS and MS-related clinical factors, including DMD use in fathers, are not associated with adverse birth outcomes $[12,13]$.

As with cladribine tablets, the majority of DMDs (dimethyl fumarate, teriflunomide, fingolimod, alemtuzumab, natalizumab, and ocrelizumab) carry a prescribing information warning that exposure during pregnancy may cause fetal harm or spontaneous abortion, based on results from animal studies [17-28]. The precise nature of the concern varies between agents. No evidence of reproductive toxicity or malformations was observed in pregnant women exposed to glatiramer acetate $[22,29]$. Recent data on pregnancy and infant outcomes from the European IFN- $\beta$ Pregnancy Registry and population-based registers in Finland and Sweden showed no evidence that IFN- $\beta$ exposure before conception and/or during pregnancy adversely affected pregnancy or infant outcomes [30]. Indeed, these findings are now reflected in the current labeling for IFN- $\beta$, which also states that IFN- $\beta$ may be considered during pregnancy if clinically needed [31-35]. For dimethyl fumarate, fingolimod, and teriflunomide, there is evidence of reproductive toxicity in animals with higher dose exposures but no clear signal, based on limited clinical experience, in human pregnancy. A small study of leflunomide (the parent compound of teriflunomide, marketed in the USA since 1998 for treatment of rheumatoid arthritis), for example, noted no substantially increased risk of adverse pregnancy outcomes due to leflunomide exposure [36]. An analysis of the global pharmacovigilance database did not indicate any teratogenic signals in patients treated with teriflunomide [37], and prospective data are being captured worldwide in the post-marketing setting. For the monoclonal antibodies natalizumab, alemtuzumab, and ocrelizumab, there is evidence of reproductive toxicity in animals; however, limited data are available on outcomes for pregnant women [23, 25-28, 38-42]. The European Public Assessment Report for Ocrelizumab detailed 6/48 cases of abnormal findings (including structural malformation, infection, and low birth weight) in live births from female subjects who participated in clinical studies, where conception occurred from approximately 3 months up to 3 years after the last administered dose [43].

A key factor that differentiates cladribine tablets from most of the other DMD treatments is the pulsed nature of administration of the drug compared with the long-term administration of maintenance therapies. Cladribine has a short half-life and is therefore eliminated from plasma and tissues less than 1 week after completion of each annual administration [6]. While there are currently no data to evaluate the interaction between cladribine and oral contraceptives, the potential for drug-drug interactions is, in principle, reduced by the short treatment schedule and exposure. However, this remains a topic of interest and a drug-drug interaction study between cladribine tablets and ethinyl estradiol plus levonorgestrel is ongoing (NCT03745144). As per product labeling, women of childbearing potential, using systemically acting hormonal contraceptives, should add a barrier method during cladribine treatment and up to 4 weeks after the last dose because of the unknown effects on hormonal birth control [5].

The effect of cladribine on human fertility is unknown [5]. In mice, there were no effects on fertility in either female or male mice; however, testicular effects were observed in mice and monkeys. Similar to female patients, as per prescribing information, male patients must take precautions to prevent pregnancy of their partner during treatment with cladribine tablets and for at least 6 months after the last dose [5].

The findings of the current analysis are limited by the small number of exposed pregnancies although the findings represent the totality of experience from the clinical development program for cladribine in MS, and will therefore provide relevant information for patients and healthcare providers alike. It is also important to consider that the present analysis was not a formal pregnancy exposure study; rather, the analysis utilized data collected through pregnancy report forms and parent child/fetus adverse event report forms used in the clinical trials. Consequently, further information relevant to pregnancy outcomes, such as dose response, timing of pregnancy, and confounders, was limited. For example, there was no information on specific patient behaviors related to risk management that may have confounded the results, such as smoking, alcohol consumption, and drug abuse during pregnancy. The present study was also limited in terms of systematic follow-up after birth, considering that 
some congenital anomalies may not be evident at the time of birth and only diagnosed at a later date. Further study is clearly warranted to provide additional information on pregnancy outcomes among cladribine-treated patients.

The clinical and radiological efficacy of cladribine 3.5$\mathrm{mg} / \mathrm{kg}$ tablets persists in many patients for 4 years or possibly longer [4]. This may potentially represent a window of opportunity for patients who want to have a child and who are willing to delay pregnancy for at least 6 months after completion of the second-year treatment course (ensuring they receive the full recommended dose for cladribine tablets). However, for all therapies used in MS, large and wellpowered studies with adequate numbers of exposed pregnancies are required to provide patients desiring pregnancy with better information on which to make their treatment decisions. In the case of cladribine tablets, CLEAR (EU PAS Register number EUPAS25027) is a non-interventional post-authorization safety study based on secondary use of data from various automated healthcare databases and registries. This is ongoing in several countries to obtain data on the effect of oral cladribine exposure, in a cohort of pregnant women with MS and in a cohort of pregnant women whose pregnancy is fathered by men with MS exposed to oral cladribine. Exposed cohorts will be compared to unexposed cohorts.

\section{Conclusions}

As MS is most commonly diagnosed in women, many of whom may be of childbearing age, reproductive planning with respect to MS treatment requires careful consideration, especially because the continuation of many DMDs is not advised during pregnancy. It is therefore important to collect and report data on pregnancy outcomes in women who have been taking DMDs or who are the partners of men with MS who have received such treatment. While limited by the small number of pregnancies and related data, highlighting the need for further study, the observations in this integrated safety analysis of the clinical development program of cladribine were consistent with epidemiological data on pregnancy outcomes for the general population or women with MS. No congenital malformations were observed with cladribine during the 'at-risk' period. It should be noted that the period of therapeutic effectiveness that patients might expect following treatment with cladribine tablets is substantially longer than the period during which recommended contraception should be practiced. However, the data currently available for pregnancy and cladribine in MS are limited, and as recommended by regulatory authorities, additional studies are needed, and a post-authorization safety study is therefore ongoing.
Acknowledgements The authors thank patients and their families; investigators; co-investigators; the study teams at each of the participating centers and at Merck KGaA, Darmstadt, Germany; and Magnhild Sandberg Wollheim for an insightful review of the draft manuscript. Medical writing assistance was provided by Sarah Wetherill and Steve Winter of inScience Communications, Springer Healthcare Ltd, Chester, UK, and was funded by Merck KGaA, Darmstadt, Germany.

Author Contributions GG, AG, RS, TL, GC, XM, DD, FD, and SC all made substantial contributions to the concept and design, or analysis and interpretation of data, and to the drafting of the manuscript or revising it critically for important intellectual content. In addition, all authors provided final approval of the manuscript.

Data Availability For all new products or new indications approved in both the European Union and the USA after 1 January, 2014, Merck $\mathrm{KGaA}$ will share patient- and study-level data after deidentification, as well as redacted study protocols and clinical study reports from clinical trials in patients. These data will be shared with qualified scientific and medical researchers, upon a researcher's request, as necessary for conducting legitimate research. Such requests must be submitted in writing to the company's data sharing portal and will be internally reviewed regarding criteria for researcher qualifications and legitimacy of the research purpose.

\section{Compliance with Ethical Standards}

Funding The design of this study and the collection and analysis of data, and medical writing and editorial support in development of this manuscript were sponsored by EMD Serono, Inc., a business of Merck KGaA, Darmstadt, Germany (in the USA), and Merck Serono SAGeneva, an affiliate of Merck KGaA, Darmstadt, Germany (rest of the world).

Conflict of interest Gavin Giovannoni has received speaker honoraria and consulting fees from AbbVie, Actelion, Atara Bio, Almirall, Bayer Schering Pharma, Biogen Idec, FivePrime, GlaxoSmithKline, GW Pharma, Ironwood, Merck \& Co., Merck KGaA, Novartis, Pfizer Inc., Protein Discovery Laboratories, Teva Pharmaceutical Industries Ltd, Sanofi-Genzyme, UCB, and Vertex Pharmaceuticals; and has received research support unrelated to this study from Biogen Idec, Ironwood, Merck \& Co., and Novartis. Andrew Galazka is an employee of Merck, Aubonne, Switzerland, a division of Merck KGaA, Darmstadt, Germany. Regina Schick is an employee of Merck KGaA, Darmstadt, Germany. Thomas Leist has received consultancy fees or clinical research grants from Acorda, Bayer, Biogen, Daiichi, EMD Serono, Novartis, ONO, Pfizer, and Teva Neuroscience. Giancarlo Comi has received consulting fees from Bayer Schering, Biogen Idec, Genentech-Roche, Merck, Novartis, Receptos, Sanofi-Aventis, and Teva Pharmaceutical Industries Ltd; lecture fees from Bayer Schering, Biogen Dompè, Merck, Novartis, Sanofi-Aventis, Serono Symposia International Foundation, and Teva Pharmaceutical Industries Ltd; and trial grant support from Bayer Schering, Biogen Dompè, Biogen Idec, Genentech-Roche, Merck, Novartis, Receptos, Sanofi-Aventis, and Teva Pharmaceutical Industries Ltd. Xavier Montalban has received speaking honoraria and travel expenses for scientific meetings, has been a steering committee member of clinical trials or participated in advisory boards of clinical trials in the past years with Actelion, Bayer, Biogen, Celgene, Excemed, Genzyme, Merck, MSIF, NMSS, Novartis, Roche, SanofiGenzyme, and Teva Pharmaceuticals. Doris Damian and Fernando Dangond are employees of EMD Serono Research \& Development Institute, Inc., a business of Merck KGaA, Darmstadt, Germany. Stuart Cook has received honoraria for lectures/consultations from Actinobac Biomed, Inc., Bayer HealthCare, Biogen Idec, Merck KGaA, Neurol- 
ogy Reviews, Sanofi-Aventis, and Teva Pharmaceuticals; has served on advisory boards for Actinobac Biomed, Inc., Bayer HealthCare, Biogen Idec, Merck KGaA, and Teva Pharmaceuticals; and received grant support from Bayer HealthCare.

Ethics approval No formal ethics approval was required in this analysis: for each clinical trial included in this integrated analysis, approval was granted by an independent ethics committee.

Consent to participate All patients provided written informed consent prior to enrollment in the clinical trial.

Open Access This article is licensed under a Creative Commons Attribution-NonCommercial 4.0 International License, which permits any non-commercial use, sharing, adaptation, distribution and reproduction in any medium or format, as long as you give appropriate credit to the original author(s) and the source, provide a link to the Creative Commons licence, and indicate if changes were made. The images or other third party material in this article are included in the article's Creative Commons licence, unless indicated otherwise in a credit line to the material. If material is not included in the article's Creative Commons licence and your intended use is not permitted by statutory regulation or exceeds the permitted use, you will need to obtain permission directly from the copyright holder. To view a copy of this licence, visit http://creativecommons.org/licenses/by-nc/4.0/.

\section{References}

1. Kamm CP, Uitdehaag BM, Polman CH. Multiple sclerosis: current knowledge and future outlook. Eur Neurol. 2014;72(3-4):132-41.

2. Montalban X, Gold R, Thompson AJ, Otero-Romero S, Amato MP, Chandraratna D, et al. ECTRIMS/EAN guideline on the pharmacological treatment of people with multiple sclerosis. Eur J Neurol. 2018;25(2):215-37. https://doi.org/10.1111/ene.13536.

3. Giovannoni G, Comi G, Cook S, Rammohan K, Rieckmann P, Soelberg Sorensen P, et al. A placebo-controlled trial of oral cladribine for relapsing multiple sclerosis. N Engl J Med. 2010;362(5):416-26. https://doi.org/10.1056/NEJMoa0902533.

4. Giovannoni G, Soelberg Sorensen P, Cook S, Rammohan K, Rieckmann P, Comi G, et al. Safety and efficacy of cladribine tablets in patients with relapsing-remitting multiple sclerosis: Results from the randomized extension trial of the CLARITY study. Mult Scler. 2018;24(12):1594-604. https://doi.org/10.1177/13524 58517727603

5. Merck Europe B.V. Mavenclad $10 \mathrm{mg}$ summary of product characteristics. 2020. Available from: www.ema.europa.eu. Accessed 13 Jan 2020.

6. Savic RM, Novakovic AM, Ekblom M, Munafo A, Karlsson MO. Population pharmacokinetics of cladribine in patients with multiple sclerosis. Clin Pharmacokinet. 2017;56(10):1245-53. https://doi.org/10.1007/s40262-017-0516-6.

7. Hartung HP, Aktas O, Kieseier B, Giancarlo Comi GC. Development of oral cladribine for the treatment of multiple sclerosis. J Neurol. 2010;257(2):163-70. https://doi.org/10.1007/s0041 5-009-5359-0.

8. Cook S, Leist T, Comi G, Montalban X, Giovannoni G, Nolting A, et al. Safety of cladribine tablets in the treatment of patients with multiple sclerosis: an integrated analysis. Mult Scler Relat Disord. 2019;29:157-67. https://doi.org/10.1016/j.msard.2018.11.021.

9. Leist TP, Comi G, Cree BA, Coyle PK, Freedman MS, Hartung HP, et al. Effect of oral cladribine on time to conversion to clinically definite multiple sclerosis in patients with a first demyelinating event (ORACLE MS): a phase 3 randomised trial.
Lancet Neurol. 2014;13(3):257-67. https://doi.org/10.1016/s1474 $-4422(14) 70005-5$.

10. Montalban X, Leist TP, Cohen BA, Moses H, Campbell J, Hicking $\mathrm{C}$, et al. Cladribine tablets added to IFN-beta in active relapsing MS: the ONWARD study. Neurol Neuroimmunol Neuroinflamm. 2018;5(5):e477. https://doi.org/10.1212/nxi.0000000000000477.

11. Pecori C, Giannini M, Portaccio E, Ghezzi A, Hakiki B, Pasto $\mathrm{L}$, et al. Paternal therapy with disease modifying drugs in multiple sclerosis and pregnancy outcomes: a prospective observational multicentric study. BMC Neurol. 2014;14:114. https://doi. org/10.1186/1471-2377-14-114.

12. Hellwig K, Haghikia A, Gold R. Parenthood and immunomodulation in patients with multiple sclerosis. J Neurol. 2010;257(4):580-3. https://doi.org/10.1007/s00415-009-5376-z.

13. Lu E, Zhu F, Zhao Y, van der Kop M, Sadovnick AD, Synnes A, et al. Birth outcomes of pregnancies fathered by men with multiple sclerosis. Mult Scler. 2014;20(9):1260-4. https://doi. org/10.1177/1352458514521308.

14. Finkelsztejn A, Brooks JB, Paschoal FM Jr, Fragoso YD. What can we really tell women with multiple sclerosis regarding pregnancy? A systematic review and meta-analysis of the literature. Br J Obstet Gynaecol. 2011;118(7):790-7. https://doi.org/10.111 1/j.1471-0528.2011.02931.x.

15. Boskovic R, Wide R, Wolpin J, Bauer DJ, Koren G. The reproductive effects of beta interferon therapy in pregnancy: a longitudinal cohort. Neurology. 2005;65(6):807-11. https://doi. org/10.1212/01.wnl.0000180575.77021.c4.

16. Ebrahimi N, Herbstritt S, Gold R, Amezcua L, Koren G, Hellwig K. Pregnancy and fetal outcomes following natalizumab exposure in pregnancy: a prospective, controlled observational study. Mult Scler. 2015;21(2):198-205. https://doi.org/10.1177/1352458514 546790.

17. EMD Serono, Inc. Rebif $22 \mu \mathrm{g}, 44 \mu \mathrm{g}$ FDA approved label. 2019. Available from: www.fda.gov. Accessed 29 Oct 2019.

18. Biogen Idec Inc. Avonex $30 \mu \mathrm{g}$ FDA approved label. 2019. Available from: www.fda.gov. Accessed 29 Oct 2019.

19. Bayer HealthCare Pharmaceuticals Inc. Betaseron 0.25 mg FDA approved label. 2019. Available from: www.fda.gov. Accessed 29 Oct 2019.

20. Novartis Pharmaceutical Corporation. Extavia $0.25 \mathrm{mg}$ FDA approved label. 2016. Available from: www.fda.gov. Accessed 11 Sep 2018.

21. Biogen Idec Inc. Plegridy $125 \mu$ g FDA approved label. 2019. Available from: www.fda.gov. Accessed 29 Oct 2019.

22. Teva Neuroscience, Inc. Copaxone $20 \mathrm{mg}, 40 \mathrm{mg}$ FDA approved label. Available from: www.fda.gov. Accessed 11 Sep 2018.

23. Biogen Inc. Tecfidera $240 \mathrm{mg}$ FDA approved label. 2017. Available from: www.fda.gov. Accessed 11 Sep 2018.

24. Genzyme Corporation. Aubagio $7 \mathrm{mg}, 14 \mathrm{mg}$ FDA approved label. 2016. Available from: www.fda.gov. Accessed 11 Sep 2018.

25. Novartis AG. Gilenya $0.5 \mathrm{mg}$ FDA approved label. 2018. Available from: www.fda.gov. Accessed 11 Sep 2018.

26. Biogen Inc. Tysabri $300 \mathrm{mg}$ FDA approved label. 2018. Available from: www.fda.gov. Accessed 11 Sep 2018.

27. Genzyme Corporation. Lemtrada $12 \mathrm{mg}$ FDA approved label. 2017. Available from: www.fda.gov. Accessed 11 Sep 2018.

28. Genentech, Inc. Ocrevus $30 \mathrm{mg} / \mathrm{mL}$ FDA approved label. 2017. Available from: www.fda.gov. Accessed 11 Sep 2018.

29. Teva Pharmaceuticals Ltd. Copaxone $20 \mathrm{mg} / \mathrm{mL}$ summary of product characteristics. 2017. Available from: www.medicines. org.uk. Accessed 11 Sep 2018.

30. Hellwig K, Duarte Caron F, Wicklein E-M, Bhatti A, Adamo A. Pregnancy outcomes from the global pharmacovigilance database on interferon beta-1b exposure. Ther Adv Neurol Disord. 2020;13. https://doi.org/10.1177/1756286420910310. 
31. Biogen Idec Ltd. Avonex $30 \mu \mathrm{g}$ summary of product characteristics. 2019. Available from: www.ema.europa.eu. Accessed 29 Oct 2019.

32. Bayer AG. Betaferon $250 \mu \mathrm{g} / \mathrm{mL}$ summary of product characteristics. 2019. Available from: www.ema.europa.eu. Accessed 29 Oct 2019.

33. Novartis Europharm Ltd. Extavia $250 \mu \mathrm{g}$ summary of product characteristics. 2019. Available from: www.ema.europa.eu. Accessed 29 Oct 2019.

34. Biogen Netherlands B.V. Plegridy $125 \mu \mathrm{g}$ summary of product characteristics. 2019. Available from: www.ema.europa.eu. Accessed 29 Oct 2019.

35. Merck Serono Europe Ltd. Rebif $22 \mu \mathrm{g}, 44 \mu \mathrm{g}$ summary of product characteristics. 2019. Available from: www.ema.europa.eu. Accessed 29 Oct 2019.

36. Chambers CD, Johnson DL, Robinson LK, Braddock SR, $\mathrm{Xu}$ R, Lopez-Jimenez J, et al. Birth outcomes in women who have taken leflunomide during pregnancy. Arthritis Rheum. 2010;62(5):1494-503. https://doi.org/10.1002/art.27358.

37. Kieseier BC, Benamor M. Pregnancy outcomes following maternal and paternal exposure to teriflunomide during treatment for relapsing-remitting multiple sclerosis. Neurol Ther. 2014;3(2):133-8. https://doi.org/10.1007/s40120-014-0020-y.

38. Biogen Idec Ltd. Tecfidera $240 \mathrm{mg}$ summary of product characteristics. 2018. Available from: www.ema.europa.eu. Accessed 11 Sep 2018.

39. Novartis Europharm Limited. Gilenya $0.5 \mathrm{mg}$ summary of product characteristics. 2018. Available from: www.ema.europa.eu. Accessed 11 Sep 2018.

40. Biogen Netherlands B.V. Tysabri $300 \mathrm{mg}$ summary of product characteristics. 2018. Available from: www.ema.europa.eu. Accessed 11 Sep 2018.

41. Genzyme Therapeutics Ltd. Lemtrada $12 \mathrm{mg}$ summary of product characteristics. 2018. Available from: www.ema.europa.eu. Accessed 11 Sep 2018.

42. Roche Registration $\mathrm{GmbH}$. Ocrevus $30 \mathrm{mg} / \mathrm{mL}$ summary of product characteristics. 2018. Available from: www.ema.europa.eu. Accessed 11 Sep 2018.

43. European Medicines Agency. Ocrevus European public assessment report. 2017. Available from: www.ema.europa.eu. Accessed 31 May 2018.

\section{Affiliations}

\section{Gavin Giovannoni ${ }^{1} \cdot$ Andrew Galazka $^{2} \cdot$ Regina Schick $^{3} \cdot$ Thomas Leist $^{4} \cdot$ Giancarlo Comi $^{5} \cdot$ Xavier Montalban $^{6,7}$. Doris Damian ${ }^{8} \cdot$ Fernando Dangond $^{8}$. Stuart Cook ${ }^{9}$}

Gavin Giovannoni

g.giovannoni@qmul.ac.uk

1 Blizard Institute, Barts and the London School of Medicine and Dentistry, Queen Mary University of London, 4 Newark Street, London E1 2AT, UK

2 Merck, Aubonne, Switzerland, a division of Merck KGaA, Darmstadt, Germany

3 Merck KGaA, Darmstadt, Germany

4 Comprehensive MS Center, Jefferson University, Philadelphia, PA, USA

5 Department of Neurology and Institute of Experimental Neurology, Università Vita-Salute San Raffaele, Ospedale San Raffaele, Milan, Italy
6 Division of Neurology, St Michael's Hospital, University of Toronto, Toronto, ON, Canada

7 Department of Neurology-Neuroimmunology, Centre d'Esclerosi Múltiple de Catalunya (Cemcat), Hospital Universitario Vall d'Hebron, Barcelona, Spain

8 EMD Serono Research and Development Institute, Inc., Billerica, MA, USA

9 New Jersey Medical School, Rutgers, The State University of New Jersey, Newark, NJ, USA 